\title{
La vie oscillatoire
}

\author{
Au coenr des rythmes \\ du vivant \\ Albert Goldbeter \\ Jacques Haiech
}

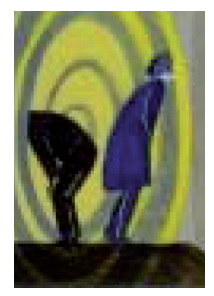

J. Haiech : École supérieure
de biotechnologie de Strasbourg,
Faculté de pharmacie,
74, route du Rhin, BP 24,
67401 IIlkirch Cedex, France.
jacques.haiech@unistra.fr

Albert Goldbeter est professeur à l'Université libre de Bruxelles et responsable de l'unité de chronobiologie théorique. Son dernier livre, La vie oscillatoire [1], est à la fois le témoignage d'une vie, un essai de compréhension du vivant et l'illustration d'une démarche scientifique.

L'hypothèse qui structure le livre est qu'aux différents niveaux d'organisation du vivant (de la molécule à la société), les phénomènes oscillants avec qu'Albert Goldbeter explore en visitant les rythmes du vivant. En nous proposant une approche qui combine modélisation théorique et validation expérimentale, il donne aux lecteurs non seulement la carte de l'espace que nous explorons mais aussi les outils et les méthodes pour voyager dans cet espace.

Le voyage se fait dans le temps et dans la complexité : dans le temps, en commençant en 1957 avec l'observation princeps des oscillations glycolytiques dans le des périodes allant de la milliseconde à plusieurs dizaines d'années permettent l'émergence de la complexité. Les variations périodiques de phénomènes biologiques créent les rythmes du vivant. Albert Goldbeter nous invite à un voyage au cœur des rythmes des vivants parfois aride quand nous explorons le niveau moléculaire, souvent poétique, quand nous abordons la cellule, l'organe, l'organisme et la famille.

Un oscillateur est un système qui évolue de part et d'autre d'une position d'équilibre. L'exemple classique de l'oscillateur est le balancier de nos vieilles horloges. $\mathrm{Ne}$ dit-on pas que l'horloge est

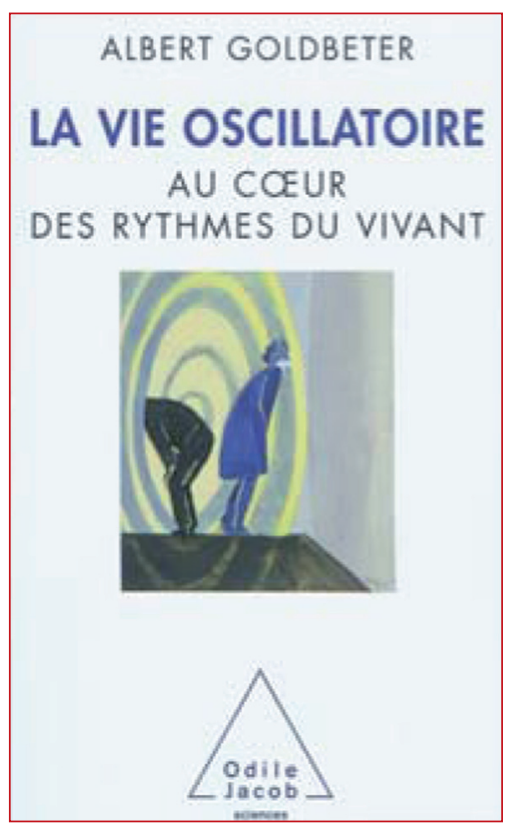

système simple de la levure, dans la complexité en présentant des rythmes à composante psychologique dans le pénultième chapitre de l'ouvrage. Après avoir présenté des rythmes isolés, il restait à Albert Goldbeter à être le chef d'orchestre de ces rythmes et à montrer leurs imbrications, c'est l'objet du dernier chapitre.

Comment avoir des phénomènes oscillants? Le livre ne présente pas une liste exhaustive des oscillateurs existant dans les différents niveaux d'organisation du vivant mais par le biais d'exemples et par petites touches successives, il fait découvrir aux lecteurs les principes nécessaires pour avoir des systèmes oscillants. Dans le cas de l'oscillation glycolytique, l'enmorte quand le balancier est arrêté, c'est-à-dire immobile dans sa position d'équilibre? Le vivant ne serait qu'un ensemble d'oscillateurs permettant d'échanger énergie, matière et information dans un même niveau ou entre niveaux d'organisation? C'est cette hypothèse zyme au cœur du système (la phosphofructokinase, PFK) est contrôlée positivement par le produit de la réaction qu'elle catalyse et l'interaction enzyme-produit exhibe une liaison coopérative. Ainsi, cet oscillateur nécessite un rétrocontrôle positif et une interaction allostérique. 
Jacques Monod, en mettant au cœur du vivant l'information et l'allostérie, avait eu probablement l'intuition que ces phénomènes favorisaient l'apprentissage de la complexité et donnait au vivant une plasticité permettant de s'adapter au contexte. Albert Goldbeter nous montre que ces propriétés sont à la base des rythmes du vivant et que la vie existe parce que nous oscillons.

Dans les organismes vivants, on trouve des oscillateurs construits avec des enzymes, des gènes ou des vésicules. D’un point de vue conceptuel, le vivant peut utiliser au moins trois langages de programmation. La compréhension de ces langages permet alors de créer de nouveaux oscillateurs ou, plus généralement, de nouveaux systèmes de traitement de l'information. On entre ainsi dans le domaine de la biologie synthétique à peine abordée dans le livre mais dont on pressent les énormes potentialités.

La lecture du livre nécessite un socle de connaissances en biologie, socle que le lycée devrait apporter pour construire l'honnête homme du $x_{x l} I^{e}$ siècle. Pour ma part, j'utiliserai aussi ce livre dans mon enseignement universitaire ; chaque chapitre peut constituer le départ d'un projet d'étude que l'on peut donner à un groupe d'étudiants en master. $\diamond$

The oscillatory life, in the heart of alive rhythms

\section{CONFLIT D'INTÉRÊTS}

L'auteur déclare n'avoir aucun conflit d'intérêts concernant les données publiées dans cet article.

\section{RÉFÉRENCE}

1. Goldbeter A. La vie oscillatoire: au cœur des rythmes du vivant. Paris : Odile Jacob, 2010 : 368 p.

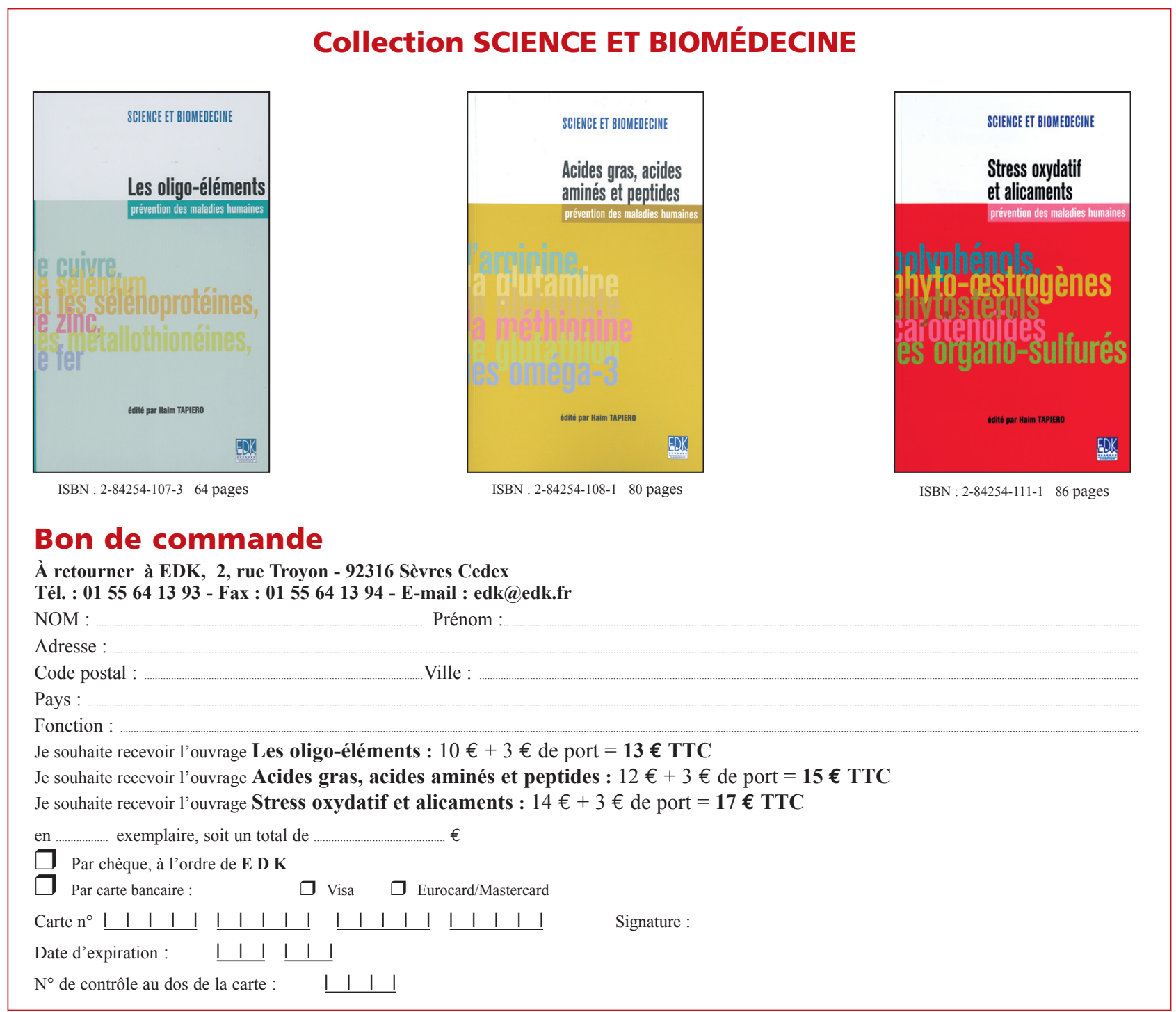

TIRÉS À PART

J. Haiech 